\title{
"Vital testimonio confirmó...": Polarización de fuentes y redes en el Caso Catrillanca en Twitter
}

\author{
"A Vital testimony confirmed...": Sources and Networks \\ Polarization over Catrillanca's Case on Twitter
"Vital testemunha confirmou...": Polarização de fontes e redes no Caso Catrillanca no Twitter

MarceloLuis Barbosa dos Santos, Universidad Finis Terrae, Santiago, Chile (msantos@uft.cl) Oscar Jaramillo Castro, Universidad Finis Terrae, Santiago, Chile (ojaramillo@uft.cl) Daniel Aguirre Azócar, Universidad del Desarrollo, Santiago, Chile (doaguirre@udd.cl)

RESUMEN | Este estudio analiza la polarización de las opiniones en Twitter que se generaron en torno al asesinato del comunero mapuche Camilo Catrillanca en Chile, en 2018, tras revelarse las irregularidades del procedimiento policial en torno a su muerte. Se explora la presencia de las llamadas cámaras de eco y las burbujas de filtro en clústeres formados por grupos con mayor presencia de usuarios radicalizados políticamente de acuerdo con sus perfiles en la plataforma. Apoyado en técnicas de análisis de redes sociales (ARS), analítica de medios sociales y análisis de contenido, se observa que las redes formadas por actores más radicales de derecha son más homofílicas y usan menor diversidad de fuentes. Se discuten los resultados, se señalan limitaciones y posibles caminos futuros.

PALABRAS CLAVES: Mapuche; Catrillanca; polarización; redes sociales; contenido generado por usuario; desinformación; Twitter; ARS.

Santos, M. L. B., Jaramillo Castro, O. \& Aguirre, D. (2021). "Vital testimonio confirmó...”: Polarización de fuentes y redes en el Caso Catrillanca en Twitter. Cuadernos.info, (49), 26-50.

https://doi.org/10.7764/cdi.49.27509 
ABSTRACT | The murder in 2018 of Camilio Catrillanca, a member of a Mapuche tribe located in the South of Chile, garnered notorious relevancy because of the event itself but also due to the failed attempt by Chilean police to manipulate evidence that pointed to police violence and abuse. This study explores networked behavior surrounding Catrillanca's death once the police coverup became public, seeking to identify echo chambers and filter bubbles within politically radicalized user clusters. Through social network analysis (SNA), social media analytics, and content analysis, the study concludes that networks on the right of the political spectrum are homophilic and refer to less diverse sources of information, pointing to network behavior resembling echo chambers and filter bubbles. The final portion of this study provides further discussion of its results in context, limitations, and future research pathways.

KEYWORDS: Mapuche; Catrillanca; polarization; social media; user-generated content; disinformation; Twitter; SNA.

RESUMO|Este estudo analisa a polarização das opiniões no Twitter que se geraram em torno do assassinato do comuneiro mapuche Camilo Catrillanca no Chile, em 2018, após a revelação das manipulações do procedimento policial na sua morte. Explora-se a presença das chamadas câmaras de eco e as bolhas de filtro em clusters formados por grupos com maior presença de usuários mais radicais politicamente segundo seus perfis na plataforma. Apoiado em técnicas de análise de redes sociais (ARS), analítica de mídia social e análise de conteúdo, se conclui que as redes formadas por atores mais radicais de direita são mais homofílicas e usam menor diversidade de fontes. Analisam-se os resultados e apontam-se limitações e possíveis caminhos futuros.

PALAVRAS-CHAVE: Mapuche; Catrillanca; polarização; redes sociais; conteúdo gerado por usuário; desinformação; Twitter; ARS. 


\section{INTRODUCCIÓN}

El estudio de la polarización política en los flujos de opinión que circulan en las redes sociales y sus consecuencias ha cobrado relevancia en el último tiempo. Este fenómeno comunicacional ha sido asociado con las burbujas de filtro y cámaras de eco, así como con tendencias a la homofilia, que son observables en las redes. Los métodos computacionales son importantes aliados para explorar estas prácticas de comunicación que generan un importante volumen de datos.

Algunos eventos sociales, como el caso del asesinato del comunero mapuche Camilo Catrillanca en Chile en 2018 y la conversación pública en Twitter generada en torno a la verdad sobre su muerte constituyen hitos para ilustrar problemáticas en el campo de la comunicación, en que la vida democrática se ve potencialmente afectada por fenómenos como la polarización política y la desinformación (Garrett et al., 2013; Vaccari, 2018). Este estudio explora la dinámica de polarización y desinformación, a partir de una nota de prensa ampliamente difundida en Twitter: "Vital testimonio confirmó que Camilo Catrillanca participó en violento robo previo a su muerte" (Crónica Chile, 2018). También se exploran otras dinámicas de conexión y comunicación en torno a la revelación de los procedimientos de Carabineros de Chile.

En el ámbito específico de la comunicación digital, desde el advenimiento de los buscadores y blogs ya se discuten metáforas que parecieran ser propias de Internet, como las burbujas de filtro (Pariser, 2011) y las cámaras de eco (Sunstein, citado por Bruns, 2019), metáforas que buscan dar explicación a fenómenos supuestamente derivados de la integración de estas tecnologías a la vida cotidiana, al quehacer informativo y a la comunicación política. Bruns $(2017,2019)$ propone que las cámaras de eco se refieren a patrones de conexión entre los usuarios, y las burbujas de filtro a prácticas de comunicación en las plataformas digitales:

Se crea una cámara de eco donde un grupo de participantes elige conectarse preferentemente entre sí, con exclusión de los externos (...) Una burbuja de filtro emerge cuando un grupo de participantes, independiente de las estructuras de red subyacentes de sus conexiones con otros, elige comunicarse preferentemente entre sí, con exclusión de los extraños (Bruns, 2017, p. 3 , énfasis en el original).

Pese la prolífica especulación sobre cómo los procesos de polarización conllevan fenómenos de aislamiento político e informacional, falta avanzar en investigaciones para obtener una mayor evidencia científica sobre las burbujas de filtro y las cámaras de eco, para evitar, como afirma Bruns $(2017,2019)$ quedarse en especulaciones de escasa definición, basadas en evidencias anecdóticas o en metáforas. El mismo autor critica la popularización de ambas expresiones vinculadas con el contexto de 
los medios digitales, limitadas a su aura de tecno-determinista, que presentan a las redes más bien como un "conveniente chivo expiatorio tecnológico" (Bruns, 2019, p. 2).

Desde la perspectiva empírica, aunque con definiciones y umbrales variados por ejemplo, cuán cerrada debe ser una red de comunicación para ser considerada una burbuja-, los estudios han sistemáticamente desestimado la asociación de ambas perspectivas con el advenimiento de las tecnologías de comunicación digitales, sean los blogs, los motores de búsqueda, los algoritmos de redes sociales digitales u otros (Adamic \& Glance, 2005; Bruns, 2017, 2019; Duggan \& Smith, 2016; Gentzkow \& Shapiro, 2011).

En este sentido, en este trabajo se busca suplir parcialmente demandas identificadas por Tucker y sus colegas (2018) al estudiar un caso de polarización y exposición a desinformación fuera de los Estados Unidos y a la vez "evaluar el rol de asimetrías ideológicas mediando el efecto de la exposición a la desinformación y a la polarización" (p.7). Adicionalmente, Bruns (2017) sostiene que se necesitan mejores evaluaciones empíricas sobre la relevancia y el impacto concreto de ambos conceptos en cuestión.

Otro fenómeno que en las investigaciones se relaciona con la problemática comunicacional de la polarización política y con su estudio específico en las redes sociales es el de la homofilia. En sociología, homofilia se puede definir ampliamente como "la tendencia a que las relaciones sociales vinculen a personas socialmente similares" (Bargsted et al., 2020), pudiendo ser motivado por diferentes factores sociales, como la edad, la religión y la posición política. Si por un lado la homofilia favorece la cohesión social, por otro puede generar distancia entre grupos sociales cohesionados entre sí, fragmentando el tejido social que, en situaciones de conflicto social agudo, puede eventualmente llevar a la polarización política (Bargsted et al., 2020). Adicionalmente, al haber liderazgos que descalifican o invisibilizan al otro, dicha polarización se puede convertir en demonización y generar el caldo de cultivo, a partir de lo simbólico, para el enfrentamiento y la violencia (RomeroRodríguez et al., 2015).

En Twitter, específicamente, se pueden conformar grupos bastante desconectados entre sí en torno a figuras de extremos ideológicos opuestos. En el caso de Chile, Alcatruz (2018) examina la polarización política en Twitter, en torno a candidatos presidenciales específicos que en su discurso apelan a la polarización, pero también en torno al debate presidencial transmitido a todo el país por la asociación de canales de televisión de Chile, la ANATEL, Asociación nacional de televisión, con escasos puentes entre los grupos, aun cuando las redes sean formadas ad-hoc (Bruns \& Burgess, 2011) en torno a una etiqueta compartida. En otras palabras, pocos o ningún individuo está vinculado a ambos polos de 
discusión, lo cual favorece la idea de la cámara de eco. Aruguete (2019) argumenta que los algoritmos de Twitter "educan a la cámara de eco, consolidan la segregación homofílica y apuntalan la polarización" (p.24).

Asimismo, no necesariamente todos los grupos sociales o incluso grupos radicales de orientación políticas distintas operan de la misma forma en sus interacciones en red. Faris y sus colegas (2017) han demostrado cómo en las elecciones estadounidenses de 2016, que ganó Donald Trump, hubo diferencias relevantes en los ecosistemas de medios consultados por los partisanos de ambos lados del espectro político. La derecha radical en los Estados Unidos estaba menos expuesta a fuentes que los autores consideran confiables, las que en principio debieran contribuir a balancear los contrapuntos, corroborar información o desmentir desinformación: "el ecosistema de medios de derecha al ser más aislado era susceptible a la propaganda y desinformación sostenida de la red" (Faris et al., 2017, p. 16). Barberá y sus colegas (2015) encontraron algo similar en un análisis de 150 millones de tuits: los liberales tenían mayor probabilidad de exponerse a fuentes de ideología opuesta que los conservadores, pero a la vez el grado de homofilia ideológica depende del tópico discutido. Los autores concluyen que "posiblemente trabajos anteriores sobreestimaron el grado de segregación ideológica en el uso de social media" (Barberá et al., 2015, p. 1). En línea con dicho estudio, Dubois y Blank (2018) relativizan el alcance de las cámaras de eco en contextos de highchoice media environments, identificando interés político y dieta diversa de medios como factores moderadores de dicho fenómeno.

Según Dalton (2008), para haber polarización del sistema político, debiera haber un número significativo de partidos en los extremos políticos. Pese a que en Chile esto último no se verifica en la actualidad, entre 1990 y 2017 hay indicios de un creciente distanciamiento ideológico generacional entre adultos y tercera edad y también entre individuos de diferentes niveles socioeconómicos, en que "opositores y adherentes tomaron distancia uno con otros y, además, se volvieron internamente más cohesionados" (Lindh et al., 2019, p. 116), que podría ser identificado con lo que Sunstein (2002) llama "polarización grupal", en que las posiciones del grupo, cuando hay deliberación, se radicalizan en vez de transitar hacia el centro (p. 176).

La polarización política ocurre en la actualidad en un escenario en el que en la disputa de significados de los sucesos, ya no solo la prensa oficial juega un rol como los tradicionales gatekeepers y determinantes de la agenda noticiosa, sino que los usuarios se convierten en gatewatchers, quienes "participaron en una práctica secundaria de vigilancia observando las historias cubiertas en otros medios, tanto convencionales como alternativos, y vinculados, compartidos y expandidos en estas historias en su propia cobertura" (Bruns, 2018, p. 2). 
Desde esta perspectiva, los usuarios dejan de ser consumidores pasivos de información para convertirse en creadores de contenido y en agentes impulsores de la circulación de las fuentes de información de su propia selección para alimentar sus redes digitales. En efecto, si esto ya sucedía de forma menos visible mediante conversaciones y circulación material de los medios impresos o electrónicos, en las redes sociales digitales dicho fenómeno se expande y convierte en algo observable específicamente a través de las acciones -individuales o agregadas- de los usuarios.

\section{El pueblo originario Mapuche y el caso Catrillanca}

En 2019, el informe del relator especial sobre la extrema pobreza y los derechos humanos en la Asamblea general de las Naciones Unidas expresaba que "los derechos de los pueblos indígenas son el talón de Aquiles del historial de Chile en lo que respecta a los derechos humanos en el siglo XXI" (Naciones Unidas, 2016, p. 15). La problemática del pueblo indígena Mapuche y el Estado chileno data de periodos coloniales y resurge con fuerza en la actualidad a partir de abusos policiales y violencia explosivas entre ambas partes, dejando al menos cuatro ciudadanos Mapuche muertos por las fuerzas policiales en democracia (Calfío et al., 2020).

La situación del pueblo Mapuche es escenario de disputas en diferentes ámbitos de la sociedad chilena: legislativo (leyes, políticas públicas, acuerdos como el Convenio 169 de la Organización Internacional del Trabajo1) y judicial, como la criminalización de la protesta social y la respuesta predominantemente punitiva del estado (Toledo Llancaqueo, 2007), o aún la aplicación de la ley antiterrorista en casos de reivindicación de tierras (Calfío et al., 2020). Así también el ámbito semiótico, es decir, la disputa por los significados de los sucesos entre los que participan en la formación de la opinión pública del país, cobra importancia como espacio de potencial discordia, aunque frecuentemente la prensa oficial, tanto nacional como regional, cubre la temática de forma desfavorable a los Mapuche, omitiendo a menudo la perspectiva y usando encuadres negativos cuando el sujeto Mapuche tiene posición activa en la sociedad y positivos cuando su posición es pasiva (Hudson et al., 2020).

Camilo Catrillanca Marín fue asesinado el 14 de noviembre de 2018 en la comunidad de Temucuicui, en la novena región. El comunero mapuche murió por un disparo en la cabeza, atribuido a miembros del Grupo de Operaciones Policiales Especiales de Carabineros de Chile, más conocido como Comando Jungla,

1. Establece la obligatoriedad de consultar a los pueblos originarios cuando hay medidas que los impactan. 
situación que no fue dada a conocer como versión oficial en un inicio. Si bien el 17 de noviembre la institución había declarado que no existían grabaciones audiovisuales del hecho -pese al protocolo de grabar las operaciones con cámaras de video-, dos días después el Centro de investigaciones periodísticas Ciper Chile publicó el testimonio clave de los sucesos (Sepúlveda, 2018), que comprueba que había al menos un video realizado por el carabinero que había disparado a Catrillanca en el operativo, capturado con una cámara GoPro. Así se destapó el escándalo del montaje de Carabineros, que había ocultado y falseado evidencias (Basadre \& Equipo Ciper, 2019) y contado una versión de los hechos ocurridos parcialmente inventada (Ramírez \& Sepúlveda, 2018). En los días siguientes, además del montaje comenzaron a circular otras evidencias que iban develando la verdad de los hechos ocurridos en torno al asesinato de Catrillanca. Tras las revelaciones, emergieron oposiciones politizadas en torno a la imagen de diferentes actores, tales como el gobierno, carabineros, los Mapuche, entre otros. Así, el análisis de la información que circulaba sentenciaba que "manipularon hechos, manipularon datos, manipularon información, manipularon protocolos, mintieron de manera descarada" (Calfío et al., 2020, p. 19). La muerte de Camilo Catrillanca fue finalmente definida como asesinato por el fiscal del caso (Basadre \& Equipo Ciper, 2019).

El periodo analizado para esta investigación exploratoria corresponde al flujo de opinión en Twitter tras revelarse dicho montaje, al unirse dos temas que históricamente dividen a la opinión pública chilena, el actuar de Carabineros y la situación histórica de uno de los pueblos indígenas originarios que habita en el sur de Chile, el pueblo Mapuche (en español, gente de la tierra). En ese contexto, este estudio se propuso responder a la siguiente interrogante:

¿Cómo fueron los patrones de conexión y los patrones de comunicación de los grupos identificados predominantemente con una u otra orientación política en Twitter?

\section{MÉTODO}

Para abordar la interrogante de investigación se aplicaron métodos de análisis de redes sociales (ARS), analítica de medios sociales² (Stieglitz et al., 2014) y análisis de contenido de una muestra del conjunto de datos, seleccionada mediante métodos computacionales (screening o filtrado), de forma que cada método se nutre del otro.

2. Se utilizó el software Tableau Desktop para el análisis y visualización de los datos, para complementar el NodeXL. 


\section{Datos}

Los datos fueron descargados de manera directa desde la API ${ }^{3}$ de Twitter el 24 de noviembre de 2018 a las 23:00 hrs de Chile Continental, con la versión de pago del software de ARS, NodeXL. En aquel momento, Twitter permitía descargar información por un periodo de hasta 10 días, por lo que se obtuvieron 18.472 tuits únicos entre el 14 al 24 de noviembre de 2018.

El término de búsqueda utilizado fue solamente Camilo Catrillanca, para obtener una coincidencia exacta. Se obtuvo un corpus compuesto por 25.595 relaciones o aristas y 10.028 vértices o nodos de red (cuentas de Twitter). NodeXL no solo recopila los tuits que tienen el término de búsqueda, sino que también todas las relaciones que se establecen mediante los retuits, menciones y réplicas (Hansen et al., 2019). De esa manera reproduce los hilos o conversaciones que se producen en la red social en torno a un tema específico (Hansen et al, 2019).

Una vez recopilados los datos, se procedió a realizar el análisis de clúster o comunidades propio del ARS con el software NodeXL. Luego se calcularon las distintas medidas de centralización (que se aplican a toda la red) y de centralidad (que se aplica a cada vértice en específico). Además de estas dos medidas se aplicó la herramienta de text mining de NodeXL para identificar: vértice (cuenta de Twitter) que inicia la relación (V1), el vértice que recibe la relación (V2), el tipo de relación que se produce (tuit, retuit, mención o réplica), el texto completo del tuit, el clúster (grupo) al que pertenece el vértice que inicia la relación, el clúster al que pertenece el vértice que recibe la relación, la o las URL específicas utilizadas en el tuit y el nombre del dominio empleado en el tuit, los bigramas (pares de palabras) más usados en cada clúster, la descripción de la cuenta de Twitter que hace su propio autor, su número ID y el link a la cuenta de Twitter.

Con estas bases de datos, se realizó un análisis de contenido en los vértices que inician la relación (V1), codificándolos a partir de palabras clave de su biografía de perfil como radicales de derecha y radicales de izquierda. En seguida se aplicó analítica de medios sociales para realizar el cruce entre ambos para identificar clústeres con predominancia de usuarios codificados, asumiendo de forma exploratoria que dicha predominancia permite inferir la militancia política a los demás miembros del clúster. Con base en el mismo método, se identificaron las principales fuentes de información difundidas por los grupos con diferentes orientaciones políticas, según agrupamientos (clústeres) de redes sociales. Este procedimiento permite analizar los patrones de comunicación preferente de los grupos identificados, es decir, sugerir grados de existencia de burbujas de filtro.

3. Application Programming Interface. 
Posteriormente, el análisis de modularidad de la red a través del ARS permitió examinar grados de conectividad entre clústeres, o más bien, aislamiento de ellos, lo cual podría caracterizar cámaras de eco.

Sin embargo, ante la ausencia de parámetros definidos en la literatura para distinguir claramente qué grado implica una cámara de eco o una burbuja de filtro (Bruns, 2019), unido al hecho de que el diseño metodológico es de carácter exploratorio, el foco es discutir en perspectiva comparada los clústeres, discutiendo tendencias, más que aventurar conclusiones tajantes. Además, se debe considerar que la autocalificación de las personas en sus perfiles para definir su orientación política no fue corroborada directamente con estas.

\section{Análisis de redes sociales}

El ARS (Hansen et al, 2011) es la aplicación de un campo amplio, como la ciencia de redes, al estudio de las relaciones humanas y sus conexiones. Es un método de análisis usado para describir y analizar los vínculos en las redes sociales entre entidades, incluidas las personas. El análisis de redes sociales no se preocupa del rating, sino de las relaciones sociales que se establecen y cómo fluye la información mediante las redes que se conforman tras las diferentes formas de contacto entre los individuos. Debido al foco en las estructuras de las redes, no solo se analizan los mensajes (tuits), sino que también las relaciones: retuits, menciones y réplicas.

La tabla 1 muestra el resumen del total de las relaciones que fueron descargadas para esta investigación. En total, se obtuvieron 24.593 relaciones, de las cuales 16.426 (66,8\% del total) corresponden a retuits, 6360 menciones (25,9\%), 1498 tuits $(6,1 \%)$ y 309 réplicas (1,3\%). Nótese que el número de relaciones no es igual al número de tuits, pues un tuit puede contener más de una relación: puede ser una respuesta mencionando a alguien o un retuit de un tuit que contiene menciones y así, sucesivamente.

Relación

\begin{tabular}{ccc}
\hline Retuits & 16.426 & $66,8 \%$ \\
\hline Menciones & 6360 & $25,9 \%$ \\
\hline Réplicas & 309 & $1,3 \%$ \\
\hline Tuits & 1498 & $6,1 \%$ \\
\hline Total & 24.593 & $100,0 \%$ \\
\hline
\end{tabular}

Tabla 1. Relaciones del caso Catrillanca con base en los tuits descargados desde el 21 al 24 de noviembre de 2018

Fuente: Elaboración propia. 


\section{Análisis de clúster}

Para realizar el análisis de clúster con NodeXL se utilizó el algoritmo ClausetNewman-Moore, que se basa en la modularidad para establecer la frontera entre los distintos grupos. Dicho algoritmo busca grupos de vértices que estén conectados densamente entre ellos y que posean pocas conexiones con otros grupos (Clauset et al., 2004; Mendes Rodrigues et al., 2011). En las redes humanas, es común que las personas conformen grupos marcados por la amistad, la homofilia o los lazos sanguíneos, pero "en el lenguaje del análisis de redes, los grupos (clústeres) son conjuntos de vértices densamente conectados que solo están escasamente conectados a otros conjuntos" (Hansen et al., 2019, p. 95).

En una red social como Twitter, NodeXL crea una conexión cuando un vértice (una cuenta de Twitter) retuitea, menciona, replica o marca como favorito un tuit efectuado por otra cuenta (De Nooy et al., 2005; Smith et al., 2014; Hansen et al., 2019). Cada una de estas acciones es mapeada y resulta en una relación entre dos usuarios, por ejemplo, el usuario (vértice) que menciona (V1) y el mencionado (V2). El algoritmo fue seleccionado porque maneja de manera adecuada los recursos computacionales cuando se trata de redes grandes de más de 10 mil relaciones (Hansen et al., 2019; Clauset et al., 2004).

El análisis de clúster del ARS se diferencia del análisis de conglomerados que normalmente se realiza en el campo de la estadística, ya que posee un objeto de estudio distinto (Himelboim et al., 2017). El análisis de conglomerados es un conjunto de técnicas estadísticas que se utilizan para clasificar objetos o casos en grupos homogéneos, que se denominan conglomerados o clústeres (Cea D’Ancona, 2004). La construcción de los grupos o clústeres se realiza a partir de las características o patrones de comportamiento de los casos estudiados (Uriel \& Aldás, 2005). A diferencia de los análisis de clústeres jerárquicos y no jerárquicos, que agrupan a los individuos a partir de las distancias que tienen con respecto a variables de corte antropológico o sociológico, el ARS es de carácter estructural, por lo que conforma los clústeres a partir de las conexiones que se establecen entre las personas gracias a las funcionalidades de la plataforma. En la medida en que las personas establecen muchas relaciones recíprocas entre ellas y pocas con el resto, conformarán un grupo o clúster.

Para este estudio, seleccionamos los cinco clústeres más poblados que tenían una orientación política marcada hacia uno de los polos políticos. Tal muestra da cuenta de 63,1\% del total de mensajes del set de datos y está compuesta por los clústeres 1 a 6 , excluyendo el 4, considerado de composición mixta, como veremos más adelante. 
En abordaje del ARS, el concepto de homofilia significa "la tendencia consciente o inconsciente de asociarse con personas que nos recuerdan a nosotros mismos" (Christakis \& Fowler, 2009, p. 17), apuntando así a la metáfora de las cámaras de eco. Una forma de averiguarlo, en ARS, es calculando índices de modularidad, una medida de la cualidad de agrupamiento de las subredes (Himelboim et al., 2017) que revela su nivel de hermeticidad. El rango de esta métrica varía de 1 a 0 , en donde el cero indica la ausencia de modularidad y el 1, la modularidad total o hermetismo: la inexistencia de relaciones con otros grupos.

En la sociedad chilena, donde predomina la clausura de grupo (Bargsted et al., 2020), es probable que los usuarios de redes más homofílicas se expongan menos a fuentes diversas de información, generando burbujas de filtro, en la forma como operacionalizamos para este estudio. Adicionalmente, como veremos, si un clúster es muy homogéneo (por ejemplo, comparte una baja diversidad de fuentes de información), la ausencia de conexiones con otros clústeres podría indicar una forma de cámara de eco, pues disminuye la probabilidad de exposición a posiciones políticas o fuentes de información distintas a las de su grupo.

\section{Análisis de contenido: orientación política de los clústeres}

Varios estudios han usado el contenido de la biografía de perfil en Twitter para identificar el campo al que pertenece un académico en Twitter (Vainio \& Holmberg, 2017), para clasificar tipos de usuarios según su relación con una protesta política (Santos, 2018), para estudiar el self-branding de periodistas en Twitter a partir de las palabras, links y metadatos de la biografía (Hanusch \& Bruns, 2017), o encontrar patrones de imágenes de perfil que se correlacionen con trazos de depresión o ansiedad (Guntuku et al., 2019). Otros estudios han realizado el procedimiento inverso, usando el contenido de la biografía como insumo para tipificar usuarios (Uddin et al., 2014) o detectar clústeres en torno a un enlace de una noticia del New York Times a partir de palabras reincidentes en las biografías (HerdaĞdelen et al., 2013).

En esta línea, es razonable esperar que expresiones de posicionamiento político en la biografía sean un indicador de radicalización política de un usuario de redes sociales. Si bien el acto de publicar mensajes y otras interacciones en Twitter puede tener más bien un carácter performativo (Papacharissi, 2012), al expresar algo en su biografía, el usuario está tomando una posición, si no permanente (Shima et al., 2017), al menos más duradera que sus posteos.

Para determinar la orientación política de los usuarios a partir de sus biografías, se realizó un procedimiento exploratorio de tres etapas: (i) filtrado (apoyado por computador) por palabras clave presentes en la biografía de los usuarios, relacionadas con la radicalidad política, seguido de una revisión manual de dichas biografías, 
Tabla 2. Palabras clave para preselección de los usuarios políticos radicales

Fuente: Elaboración propia. Por razones técnicas, la búsqueda automática se realizó con minúsculas.

(ii) identificación de la presencia de dichos usuarios en los clústeres mapeados previamente con ARS, como un indicador de predominio de usuarios de una u otra tendencia política, y (iii) análisis de los bigramas más mencionados en los textos de los tuits pertenecientes a cada clúster para ratificar su tendencia política identificada a partir de la predominancia de usuarios codificados como de izquierda o de derecha.

Para identificar el grupo de usuarios radicales de derecha y de izquierda, por lo tanto, se ha hecho un breve análisis de contenido con base en un grupo de palabras clave que designan radicalidad política, en la medida en la que fueron identificadas en la biografía de los usuarios de Twitter de la muestra, según la tabla 2.

De un total de 9753 usuarios emisores (V1), 1546 presentaron incidencia de las palabras clave identificadas (606 derecha y 940 izquierda). Sin embargo, es habitual que usuarios se definan mediante la negación discursiva: "Jamás por la izquierda" o "anti-Pinochet". Por esta razón se procedió a un chequeo individual manual de las descripciones, ajustando el resultado para mayor validez. En casos de ausencia de claridad, se recurrió al usuario en Twitter para observar sus mensajes recientes y evaluar su orientación política de forma definitiva. El resultado final tras dicho procedimiento fue de 1383 usuarios políticamente radicales, siendo 476 de derecha y 907 de izquierda, casi el doble de sus opuestos, mostrando un desbalance razonable entre la presencia de ambos grupos en la discusión en torno a la temática seleccionada en favor de los usuarios radicales de izquierda.

Tras estudiar la homofilia en Chile, Bargsted y sus colegas (2020) concluyen que "hay una marcada discontinuidad en el nivel de interacción entre personas con y sin preferencias ideológicas definidas" (p. 603). En otras palabras, chilenos con preferencia ideológica definida interactúan mucho menos con aquellos sin preferencia ideológica definida y viceversa. Si los usuarios sin posición política definida interactúan poco con aquellos que sí la tienen, planteamos que aquellos que sí interactúen -y, por lo tanto, sean incluidos en el clústertienen una tendencia a ser también usuarios con una posición definida, pero no declarada en su biografía. Ante la ausencia de los radicales codificados del espectro opuesto en todos los clústeres, a excepción del 4, proponemos por lo tanto extrapolar la codificación según dicha orientación, tal como se señala en la tabla 4. 
Clúster

1

2

3

4

5

6

Bigramas más frecuentes en los tuits

asesinato,camilo muerte,camilo comando,jungla repudio, asesinato

fuerzas, especiales canal,13 13, años pueblo,mapuche minuto, silencio

muerte,camilo ahora,manifestación manifestación,pacífica 100,personas

palacio,moneda pacífica,silenciosa silenciosa, muerte catrillanca,frente

frente, palacio

violento, robo vital,testimonio testimonio,confirmó confirmó,camilo catrillanca,participó participó,violento robo, previo previo, muerte renuncia,intendente

muerte,camilo asesinato,camilo minuto, silencio rostro,camilo centenar,jóvenes jóvenes, rodea rodea, moneda moneda, rostro comando,jungla

comando,jungla 1,piñera piñera,crea crea,comando jungla,2 2,comando jungla, asesina asesina,camilo catrillanca, 3

colegio,san san,ignacio ignacio,bosque catrillanca,vía bosque,bandera bandera,mapuche mapuche,media media,asta asta,señal

Por razones técnicas, la búsqueda se hizo en minúsculas.

Tabla 3. Bigramas más frecuentes en los tuits, por clúster

Fuente: Elaboración propia con base en NodeXL.

En otras palabras, los usuarios emisores (V1) de los clústeres 1, 2, 5 y 6 fueron todos codificados como Usuario-Izquierda, mientras los usuarios del clúster 3 fueron codificados como Usuario-Derecha, sin discutir la intensidad de dicha orientación. Esta maniobra de evidente carácter exploratorio permite algunos hallazgos a ser discutidos dentro de las limitaciones inherentes de dicho alcance.

Adicionalmente, se realizó un análisis de los bigramas más frecuentes para verificar tendencias políticas a partir de estas combinaciones de palabras (tabla 3). Obsérvese la diferencia entre los bigramas del clúster 3 (negativos, criminalizando a Catrillanca) versus los del 1, 2, 5 y 6, caracterizados como clústeres de izquierda (tabla 4). Cabe destacar que los bigramas del clúster 3 están casi todos relacionados con la nota de prensa más compartida titulada "Vital testimonio confirmó que Camilo Catrillanca participó en violento robo previo a su muerte" (Crónica Chile, 2018), punto que se analizará con mayor detención.

Tras la codificación de los usuarios, la muestra fue incorporada nuevamente en la base de datos para identificar su incidencia en cada clúster y analizar las fuentes usadas, apoyados por procedimientos de analítica de medios sociales. 


\section{RESULTADOS Y DISCUSIÓN}

La red de relaciones del conjunto de datos es una red relativamente modular $(0,57)$, implicando que de forma general hay poca conectividad entre los diferentes subgrupos identificados a partir del análisis de clústeres. A continuación, se observan los principales clústeres en mayor detalle y la modularidad individual de cada clúster para poder inferir algunas observaciones sobre las problemáticas abordadas en esta investigación referente a las cámaras de eco y de las burbujas de filtro.

\section{Metáfora 1: cámaras de eco}

El método aplicado resultó en seis clústeres, cinco de los cuales presentan algún grado de polarización (tabla 4) : cuatro están conformados principalmente por usuarios de izquierda (clústeres 1-2-5 y 6) y uno tiene predominancia de usuarios de derecha (clúster 3). Solo uno (clúster 4) presenta una composición que denominamos mixta en la clasificación nominal de los grupos. Se decidió, por lo tanto, excluir al clúster 4 de la suma del total para cada columna en dicha tabla para observar mejor los polos.

ESe observa que hay un cierto equilibrio relativo en esta muestra en cuanto a usuarios codificados como radicales de derecha y radicales de izquierda, pues ambos representan entre 8 y $9 \%$ del total de usuarios en los clústeres estudiados.

Sin embargo, la concentración de usuarios radicales de derecha en el clúster 3 es notable: 424 usuarios, lo cual indica que $70 \%$ de todos los usuarios codificados como radicales de derecha están concentrados en un único clúster, mientras el clúster codificado como Izquierda 1 contiene poco menos de $40 \%$ de los usuarios codificados como radicales de izquierda. En términos de un análisis general del comportamiento de la red, esto implica que, si bien la muestra tiene predominancia de usuarios que podrían ser categorizados como de izquierda, aquellos usuarios que codificamos como radicales de derecha están casi todos concentrados en un solo clúster. Asimismo, dicho clúster es el que posee la mayor modularidad $(0,95)$. Ello implica que solo $5 \%$ de los mensajes configuran conexiones con otros clústeres - para efectos de comparación, el segundo clúster más endogámico es el 1, con $77 \%$ de interacciones dentro del mismo grupo-. El clúster 3 presenta características, al menos en el carácter relativo a la red estudiada, que podrían ser asociadas a la idea de cámaras de eco, ya que las interacciones conectan poco otros clústeres que están codificados con diferentes orientaciones políticas. 


\begin{tabular}{|c|c|c|c|c|c|c|c|c|}
\hline & \multicolumn{2}{|c|}{ Derecha } & \multicolumn{2}{|c|}{ Izquierda } & \multirow[t]{2}{*}{ 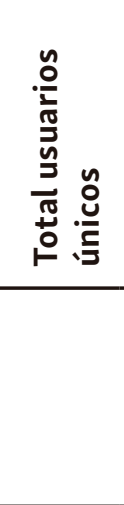 } & \multirow[t]{2}{*}{ 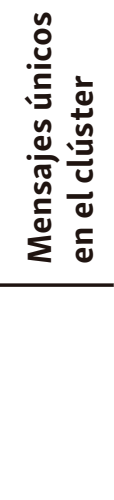 } & \multirow[t]{2}{*}{ 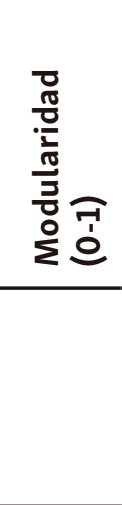 } & \multirow[t]{2}{*}{ 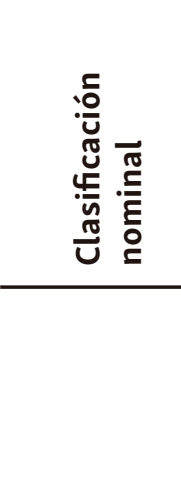 } \\
\hline & 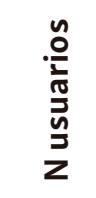 & 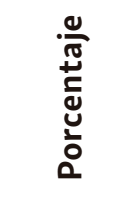 & 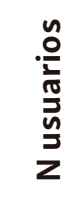 & 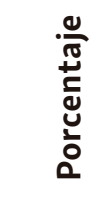 & & & & \\
\hline Clúster 1 & 2 & $0,1 \%$ & 373 & $21,6 \%$ & 1.730 & 4.245 & 0,77 & Izquierda 1 \\
\hline Clúster 2 & 1 & $0,1 \%$ & 53 & $4,3 \%$ & 1.237 & 1.428 & 0,68 & Izquierda 2 \\
\hline Clúster 3 & 424 & $34,6 \%$ & 8 & $0,7 \%$ & 1.224 & 1.944 & 0,95 & Derecha \\
\hline Clúster 4 & 11 & $1,6 \%$ & 25 & $3,6 \%$ & 693 & 1.009 & 0,61 & Mixto \\
\hline Clúster 5 & 2 & $0,3 \%$ & 27 & $4,0 \%$ & 668 & 725 & 0,57 & Izquierda 3 \\
\hline Clúster 6 & 1 & $0,2 \%$ & 17 & $3,5 \%$ & 485 & 507 & 0,66 & Izquierda 4 \\
\hline SUMA* & 430 & $8,0 \%$ & 478 & $8,9 \%$ & 5.344 & 8.849 & & \\
\hline
\end{tabular}

${ }^{*}$ Excluye Clúster 4.

Tabla 4. Análisis de los clústeres según incidencia de usuarios codificados como radical de derecha y radical de izquierda

Fuente. Elaboración propia.

\section{Metáfora 2: burbuja de filtro}

Si bien la metáfora de la burbuja de filtro nace en una observación (no sistemática) del comportamiento del buscador de Google y de sus diferentes resultados para diferentes usuarios (Pariser, 2011) - de ahí la idea de que la burbuja es provocada por un filtro de carácter algorítmico-, en este estudio no se exploran los efectos de filtros u otros tipos de interferencias algorítmicas, al menos no de forma directa.

Considerando una definición más amplia, en la que las burbujas de información se generan por los procedimientos de compartir de actores humanos y no humanos (Latour, 2004), se puede observar en los datos extraídos cuáles son los dominios de sitios más compartidos por los usuarios de los diferentes clústeres, centrándonos en el componente humano de la ecuación y buscando los patrones entre orientación política de los usuarios y las características de las fuentes que comparten en sus publicaciones. 
Un análisis general de la incidencia de dominios de Internet en los mensajes del set de datos sugiere que hay una distinción evidente en las menciones a los medios de orientación política más marcada. Por el lado de los usuarios de derecha, predomina la difusión de Crónica Chile, un diario con pautas marcadamente críticas hacia la izquierda, vinculado con grupos de la derecha libertaria como Fundación para el Progreso. Por el lado de la izquierda, se destaca El Desconcierto, un medio emergente que, a pesar de no adscribirse a ningún color partidario, declara en su línea editorial que "guarda una distancia crítica de los poderes económicos y políticos y se inscribe de forma amplia en el malestar social y las voluntades emancipadoras y de transformación de la sociedad" (El Desconcierto, s/f, par. 1). Tanto Crónica Chile como El Desconcierto tienen tasas de $99 \%$ de menciones ${ }^{4}$ por usuarios radicales de derecha e izquierda, respectivamente. El sitio de la Radio Bío Bío es el único compartido por ambos lados, ya que sus menciones se dividen en $84 \%$ de menciones por usuarios de izquierda versus $16 \%$ por usuarios de derecha. Considerando que el set de datos, tras la codificación, contiene sustancialmente más usuarios codificados como izquierda (77\%) que como derecha (23\%), esta distribución podría indicar una razonable percepción de neutralidad del medio por parte de los usuarios.

Si consideramos también los usuarios codificados como Usuario-Indefinido, es decir, todos los usuarios de los demás clústers (excepto los codificados), tenemos una distribución menos claramente marcada, pero que permite rápidamente visualizar la poca diversidad de dominios que es objeto de interacción por usuarios codificados como derecha en la muestra. Solo Crónica Chile, Bío Bío, Cooperativa y Ahora Noticias presentan cinco o más interacciones de estos usuarios en la muestra (gráfico 1).

Se operacionaliza la burbuja de filtro acá, en cuanto acto comunicativo, como la opción de interactuar con ciertas fuentes de información. En esta interpretación, una burbuja se formaría en un grupo que utiliza el mismo conjunto de fuentes, haciendo que queden menos expuestos a relatos divergentes y eventualmente incluso a fuentes de verificación de hechos (Faris et al., 2017).

Lo primero que se observa en el gráfico 2 es la predominancia de Crónica Chile y las pocas otras fuentes que lo acompañan en el clúster 3. Claramente, se constituyó un clúster radical de derecha en torno a las publicaciones de dicho medio, en particular una nota titulada "Vital testimonio confirmó que Camilo Catrillanca participó en violento robo previo a su muerte" (Crónica Chile, 2018). Este hecho en sí no debiera ser preocupante, pero un análisis más profundo muestra circunstancias que levantan una problemática mayor.

4. Este número es calculado observando solamente los dominios con los cuales los usuarios radicales tuvieron alguna interacción como autores. 


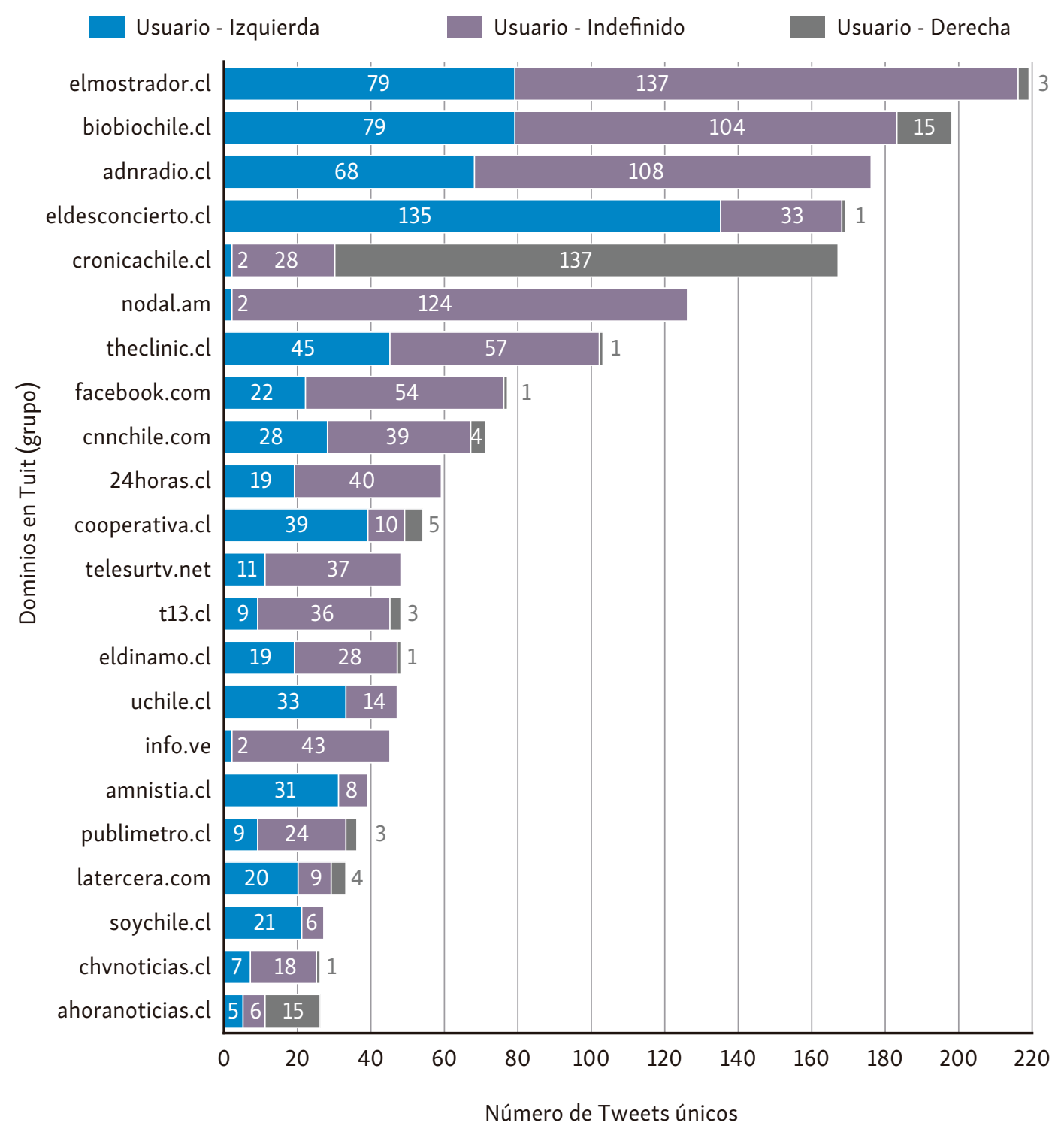

\section{Gráfico 1. Incidencia de las principales fuentes según lo difundido por usuarios catalogados como indefinido, izquierda o derecha}

Fuente: Elaboración propia.

Por un lado, dicho reportaje es específicamente un refundido de reportajes de otros medios en un momento (21/11/2018), en que las informaciones articuladas en la nota ya habían sido ampliamente cuestionadas por medios respetados como Ciper Chile.

En un clúster que ya es altamente modular ( $95 \%$ de las interacciones con otros miembros de ese clúster), las interacciones de los usuarios se dan, además, con poca diversidad de fuentes: $80 \%$ de las interacciones con fuentes se dan con Crónica Chile en el clúster 3 , mientras el clúster más radical de izquierda presenta una concentración de tan solo 32\% con El Desconcierto. 


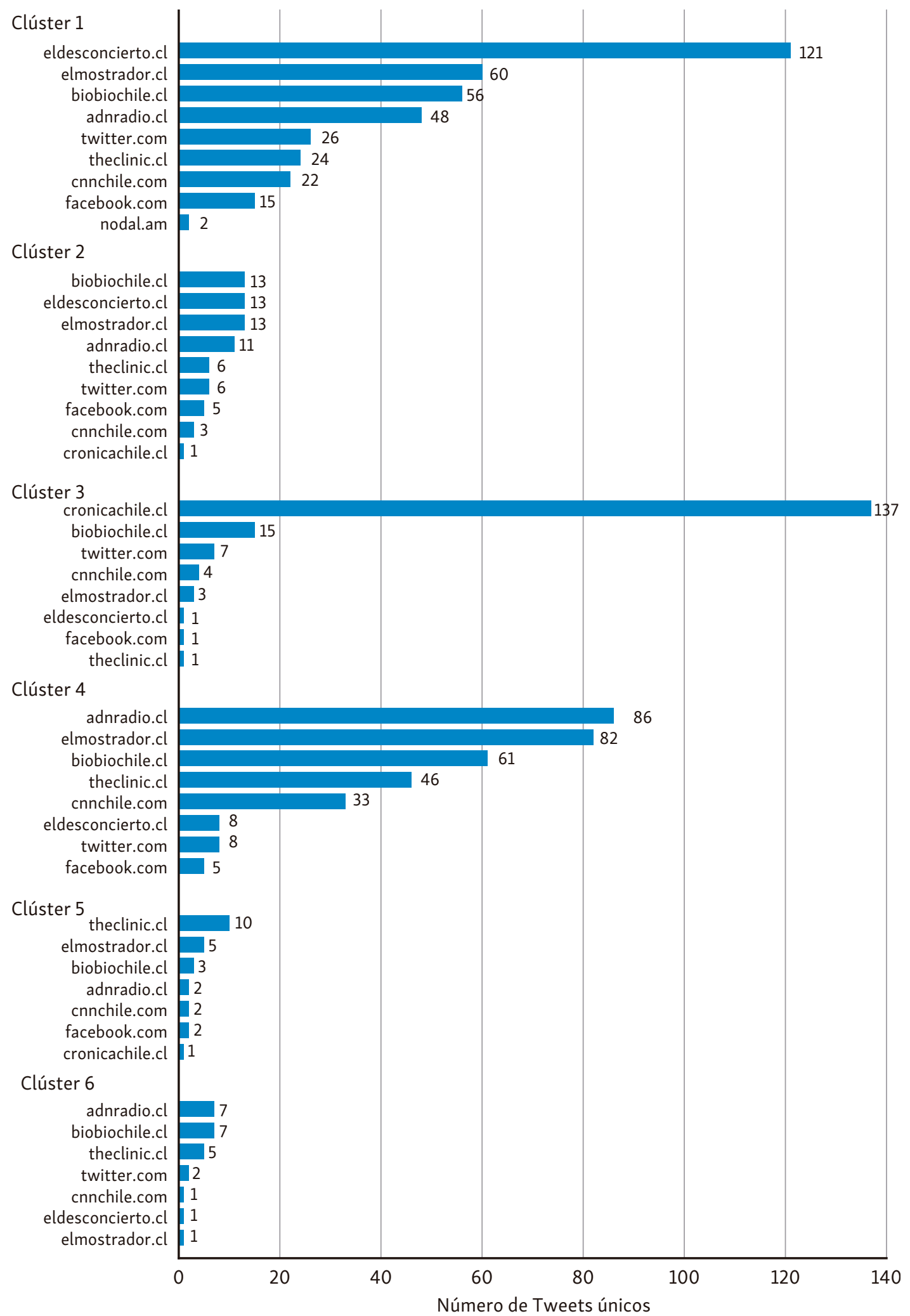

\section{Gráfico 2. Principales fuentes (según sus dominios web) citadas en las interacciones en cada clúster, en los 6 primeros clústeres}

Fuente: Elaboración propia. 
Otra evidencia que debiera apuntar a la poca confiabilidad del medio es que a menos de dos años de su publicación el artículo ya no estaba disponible en el sitio. Puede ser por razones técnicas, posiblemente la reciente actualización de interfaz del sitio, ya que pruebas con otros términos y artículos antiguos tampoco arrojan resultados. De una u otra forma, la cobertura del caso Catrillanca realizada por este sitio no estaba disponible en el momento de la escritura de este artículo.

En síntesis: se arma un clúster radical de derecha de usuarios hablando entre sí en torno a una fuente de dudosa calidad, con mínima interfaz con otras fuentes y otros usuarios fuera del grupo, configurando un caso que podría ser clasificado tanto como burbuja de filtro como de cámara de eco.

El clúster 1 sería el análogo opuesto del 3, pero muestra una diversidad de fuentes mayor. Los clústeres 2, 5 y 6 parecen no girar mucho en torno a las fuentes, por lo que probablemente son más conversacionales de opinión, como demuestran los bigramas (tabla 3). Otro clúster relevante, el 4, tiene la mayor predominancia de fuentes diversas y plurales, lo cual posiblemente explica su carácter mixto en cuanto a la orientación política de los usuarios agregados en este grupo.

A modo de síntesis, si bien los datos presentados no parecen apuntar para la existencia de burbujas de filtro en todos los casos, se puede afirmar que, en el caso estudiado, el clúster de derecha (3) presenta un grado de homofilia más intenso que todos los demás analizados e identificados con la izquierda, inclusive el clúster más notoriamente de izquierda (1). Esto permite responder parcialmente la interrogante del estudio, pues se identifica una diferencia significativa en los patrones de conexión de diferentes orientaciones políticas, en línea con Barberá y sus colegas (2015) y Faris y sus colegas (2017). Similarmente, si bien la presencia de cámaras de eco no es evidente en todos los casos, parece haber una tendencia a menor diversidad de fuentes en las interacciones de los usuarios aquí identificados con la derecha que con la izquierda.

\section{CONCLUSIONES Y PRÓXIMOS PASOS}

Aislamiento informativo, baja diversidad y pluralidad de fuentes consultadas, baja calidad de las fuentes y ausencia de diálogo con personas o fuentes de diferentes perspectivas son problemas reales en el contexto político de un país, ya sea por fuerza de dispositivos de control forzoso -como la censura- o por dispositivos sociotécnicos, como las formas de apropiarse de la tecnología. Este estudio buscó identificar tendencias y establecer posibles métodos para evaluar dichos fenómenos en el contexto de un hito viralizado en Twitter. 
En el campo del análisis de redes sociales no existe una metodología única y establecida, por lo que la mayor parte de las investigaciones, como esta, tienen un carácter exploratorio y experimental (Himelboim, et al., 2017; Mendes Rodrigues et al., 2011). El método propuesto podría servir como insumo para aproximaciones mixtas, incluyendo netnografías, análisis de contenido y otras que permitan una mirada más cualitativa a uno o dos clústeres, por ejemplo, de forma a simultáneamente profundizar los hallazgos y ratificar -o bien rectificar- el método.

Otras estrategias podrían complementar los hallazgos y precisarlos, como observar no solo fuentes que los usuarios comunican activamente, pero también fuentes consultadas, pues evidentemente no es lo mismo retuitear, seguir, leer, ponerle favorito o responder a un mensaje en Twitter. Si bien los usuarios, por su orientación política, podrían tender a amplificar solamente contenidos alineados con su visión ideológica, esto no asegura que no se informen por otros medios. De hecho, estudios apuntan que ciudadanos con orientación política radical -o hiperpartisanos- se informan en fuentes antagónicas, incluso para armarse, preparar sus argumentos, o cuestionar dichas fuentes (Garrett et al., 2013). En situaciones de conflicto en las que emerge un alto grado de polarización afectiva, los mismos detractores de un tema, medio o persona, pueden convertirse en los principales agentes de visibilización de aquello que originalmente rechazan (Santos, 2020). Adicionalmente, el campo se beneficiaría en una perspectiva ecológica de los medios (Postman, 1998; Treré \& Mattoni, 2016), abarcando diferentes medios, otras plataformas y soportes en interrelación.

Sobre las metáforas tratadas en el estudio, es importante resaltar que el hecho de que emerjan patrones que indican grados importantes de lo que se podría llamar burbuja de filtro y cámara de eco no redunda en una evidencia de que ello suceda como función exclusiva de Twitter y de sus características, por lo que evitamos una visión tecno-determinista. Los hallazgos pueden ser vistos más bien como un fenómeno probablemente vinculado con la polarización adscrita al caso y al contexto nacional que a la plataforma; a patrones sociotécnicos que a sus funcionalidades. Lo que sí nos permiten dichas plataformas es el privilegio de visualizar dichas relaciones y esto en sí es un punto positivo, no negativo, de los medios digitales, desde la perspectiva de la transparencia de los fenómenos sociales. 


\section{REFERENCIAS}

Adamic, L. A. \& Glance, N. (2005, August). The political blogosphere and the 2004 US election: divided they blog. In Proceedings of the 3rd international workshop on Link discovery (LinkKDD '05) (pp. 36-43). New York, NY: Association for Computing Machinery. https://doi.org/10.1145/1134271.1134277

Alcatruz, D. (2018, August). Polarización política en Twitter (Political polarization in Twitter) (paper presentation). XIII Congreso Chileno de Ciencia Política, Santiago, Chile. http://doi.org/10.13140/RG.2.2.27803.41768

Aruguete, N. (2019, January 22). ¿Twitter acrecienta en la Polarización Política? (Does Twitter increase political polarization?). Beers \& Politics. Retrieved from https://beersandpolitics. com/twitter-acrecienta-la-polarizacion-politica

Barberá, P., Jost, J. T., Nagler, J., Tucker, J. A., \& Bonneau, R. (2015). Tweeting from left to right: Is online political communication more than an echo chamber? Psychological Science, 26(10), 1531-1542. http://www.doi.org/10.1177/0956797615594620

Bargsted, M. A., Espinoza, V., \& Plaza, A. (2020). Pautas de homofilia en Chile (atterns of Homophily in Chile). Papers. Revista de Sociologia, 105(4), 583-612. https://doi.org/10.5565/rev/papers.2617

Basadre, P. \& Equipo Ciper. (2019, February 2). Muerte de Catrillanca: así se inventó la versión falsa de Carabineros (Catrillanca's death: this is how the false police version was created). Ciper Chile. https://ciperchile.cl/2019/02/01/muerte-de-catrillanca-asi-se-invento-laversion-falsa-de-carabineros/

Bruns, A. (2017, September). Echo chamber? What echo chamber? Reviewing the evidence. In 6th Biennial Future of Journalism Conference (FOJ17), 14-15 September 2017, Cardiff, United Kingdom. (Unpublished). Retrieved from https://eprints.qut.edu.au/113937/

Bruns, A. (2018). Gatewatching and news curation: Journalism, social media, and the public sphere. New York, USA: Peter Lang.

Bruns, A. (2019, July). It's not the technology, stupid: How the 'Echo Chamber' and 'Filter Bubble' metaphors have failed us. In International Association for Media and Communication Research conference, 7-11, July 2019, Madrid, Spain (unpublished).

Bruns, A. \& Burgess, J. E. (2011, August). The use of Twitter hashtags in the formation of ad hoc publics. In 6th European Consortium for Political Research General Conference, 25-27 August 2011, University of Iceland, Reykjavik.

Calfío, M., Coñuepan, V., \& Figueroa Huencho, V. (2019). Situación actual de los derechos del pueblo mapuche después del caso Catrillanca (Current situation of Mapuche people's rights in the aftermath of Catrillanca's case). Anuario de Derechos Humanos, 15(1), 15-40. http://doi.org/10.5354/0718-2279.2019.53921

Cea D’Ancona, M. A. (2004). Análisis multivariable. Teoría y práctica en la investigación social (Multivariable analysis. Theory and practice in social research). Madrid, Spain: Editorial Síntesis.

Clauset, A., Newman, M. E., \& Moore, C. (2004). Finding community structure in very large networks. Physical review. E, Statistical, nonlinear, and soft matter physics, 70(6), 066111. https://doi.org/10.1103/PhysRevE.70.066111 
Christakis, N. A. \& Fowler, J. H. (2009). Connected: The surprising power of our social networks and how they shape our lives. New York, USA: Little, Brown \& Company.

Crónica Chile (2018, November 21). Vital testimonio confirmó que Camilo Catrillanca participó en violento robo previo a su muerte (Vital testimony confirmed that Camilo Catrillanca participated in a violent robbery prior to his death). Crónica Chile. https://www.cronicachile.cl/2018/11/21/chilecorrupcion/vital-testimonio-confirmoque-camilo-catrillanca-participo-en-violento-robo-previo-a-su-muerte/

Dalton, R. J. (2008). The quantity and the quality of party systems: Party system polarization, its measurement, and its consequences. Comparative Political Studies, 41(7), 899-920. https://doi.org/10.1177/0010414008315860

De Nooy, W., Mrvar, A., \& Batagelj. (2005). Exploratory Social Network Analysis with Pajek. Cambridge, United Kingdom: Cambridge University Press.

Dubois, E. \& Blank, G. (2018). The echo chamber is overstated: The moderating effect of political interest in diverse media. Information, communication and society, 21(5), 729-745. https://doi.org/10.1080/1369118X.2018.1428656

Duggan, M. \& Smith, A. (2016). The Political Environment on Social media. Pew Research Center. Retrieved from http://assets.pewresearch.org/wp-content/uploads/sites/14/2016 /10/24160747/PI_2016.10.25_Politics-and-Social-Media_FINAL.pdf

El Desconcierto. (s/f). Línea Editorial (Editorial guideline). El Desconcierto. Retrieved from https://www.eldesconcierto.cl/linea-editorial/

Faris, R., Roberts, H., Etling, B., Bourassa, N., Zuckerman, E., \& Benkler, Y. (2017). Partisanship, Propaganda, and Disinformation: Online Media and the 2016 U.S. Presidential Election. Berkman Klein Center for Internet \& Society Research Paper. Retrieved from http://nrs.harvard.edu/urn-3:HUL.InstRepos:33759251

Garrett, R. K., Carnahan, D., \& Lynch, E. (2013). A Turn Toward Avoidance? Selective Exposure to Online Political Information, 2004-2008. Political Behavior, (35), 113-134. http://doi.org/10.1007/s11109-011-9185-6

Gentzkow, M. \& Shapiro, J. M. (2011). Ideological segregation online and offline. The Quarterly Journal of Economics, 126(4), 1799-1839. https://doi.org/10.1093/qje/qjr044

Guntuku, S. C., Preotiuc-Pietro, D., Eichstaedt, J. C., \& Ungar, L. H. (2019, July). What twitter profile and posted images reveal about depression and anxiety. In Proceedings of the international AAAI conference on web and social media (ICWSM 2019), vol. 13 (pp. 236-246). Retrieved from https://ojs.aaai.org/index.php/ICWSM/article/view/3225

Hansen, D., Shneiderman, B., \& Smith, M. (2011). Analyzing social media networks with NodeXL. Elsevier.

Hansen, D., Shneiderman, B., Smith, M., \& Himelboim, I. (2019). Analyzing Social media Networks with NodeXL: Insights from a Connected World (Second). Burlington, USA: Morgan Kaufmann Publishers.

Hanusch, F. \& Bruns, A. (2017). Journalistic branding on Twitter: A representative study of Australian journalists' profile descriptions. Digital journalism, 5(1), 26-43. https://doi.org/10.1080/21670811.2016.1152161 
HerdaĞdelen, A., Zuo, W., Gard-Murray, A., \& Bar-Yam, Y. (2013). An exploration of social identity: The geography and politics of news-sharing communities in twitter. Complexity, 19(2), 10-20. https://doi.org/10.1002/cplx.21457

Himelbomin, I., Smith, M., Rainie, L., Shneiderman, B., \& Espina, C. (2017). Classifying Twitter topic-networks using Social Network Analysis. Social Media + Society 3(1), 1-13. https://doi.org/10.1177/2056305117691545

Hudson, E., del Valle, C., \& Browne, R. (2020). Análisis de la Relevancia Informativa en la Cobertura del Tema Mapuche en la Prensa Nacional y Regional de Chile (Analysis of the informative relevance of the Mapuche issue in local and national press in Chile). In P. Valdivia \& C. Del Valle (Eds.), Leyendo el Tejido Social (Reading the social tissue) (pp. 355368). Temuco, Chile: Editora UFRO.

Latour, B. (2004). Redes que a razão desconhece: laboratórios, bibliotecas, coleções (Networks that reason does not know: laboratories, libraries, collections). In Tramas da rede: novas dimensões filosóficas, estéticas e políticas da comunicação (Network plots: new philosophical, aesthetic and political dimensions of communication) (pp. 39-63). Porto Alegre, Brazil: Sulina.

Lindh, J., Fábrega, J., \& González, J. (2019). La fragilidad de los consensos. Polarización ideológica en el Chile post Pinochet (The Fragility of Consensus: Ideological Polarization in Post-Pinochet Chile). Revista de Ciencia Política (Santiago), 39(1), 99-127. https://doi.org/10.4067/S0718-090X2019000100099

Mendes Rodrigues, E., Milic-Frayling, N., Smith, M., Shneiderman, B., \& Hansen, D. (2011). Group-in-a-Box Layout for Multi-faceted Analysis of Communities. In Proceedings of the IEEE Third International Conference on Privacy, Security, Risk and Trust and 2011 IEEE Third International Conference on Social Computing (pp. 354-361). https://www.doi.org/10.1109/PASSAT/SocialCom.2011.139

Naciones Unidas. (2016). Informe del Relator Especial sobre la extrema pobreza y los derechos humanos a la Asamblea General de las Naciones Unidas (Report of the Special Rapporteur on extreme poverty and human rights to the United Nations General Assembly). Oficina de Alto Comando Naciones Unidas Derechos Humanos. https://acnudh.org/load/2016/08/G1607251EXTREMAPO.pdf

Pariser, E. (2011). The Filter Bubble: What the Internet is Hiding from You. Penguin.

Papacharissi, Z. (2012). Without you, I'm nothing: Performances of the self on Twitter. International Journal of Communication, 6(18). Retrieved from https://ijoc.org/index.php/ijoc/article/view/1484

Postman, N. (1998, March 28). Five things we need to know about technological change. Talk delivered Conference in Denver, Colorado. Retrieved from https://web.cs.ucdavis.edu/ rogaway/classes/188/materials/postman.pdf

Ramírez, E. \& Sepúlveda, N. (2018, December 4). La reconstrucción del crimen de Catrillanca deja en evidencia que alguien ordenó mentir (The reconstruction of Catrillanca's crime shows that someone ordered to lie). Ciper Chile. Retrieved from https://ciperchile.cl/2018/12/04/lareconstruccion-del-crimen-de-catrillanca-deja-en-evidencia-que-alguien-ordeno-mentir/ 
Romero-Rodríguez, L. M., Aguaded, I., \& Gadea, W. (2015). De la demonización a la polarización: un análisis desde el discurso digital del gobierno y la oposición venezolana (From demonization to polarisation: An analysis of the Venezuelan government's and the political opposition's digital discourse). Argos, 32(62), 97-117.

Santos, M. (2018). Propuesta de conceptualización del Contenido Generado por Usuario de carácter testimonial (CGUt) en Twitter aplicada al caso de las protestas contra el impeachment de Dilma Rousseff (From Testimony to Testimonial: A proposal for the conceptualization of testimonial User-Generated Content (tUGC) on Twitter applied to the case of the protests against Dilma Rousseff's impeachment) (Doctoral Dissertation). Retrieved from https://repositorio.uc.cl/xmlui/handle/11534/22328

Santos, M. (2020). NÃO ALIMENTE O MINION!: Polarização afetiva e ativismo de rede às avessas na gênese e ascensão da hashtag\# Bolsonaro2018 após o impeachment de Dilma Rousseff (DO NOT FEED THE MINION! Affective polarization and backward network activism in the genesis and rise of the hashtag \#Bolsonaro2018 after the impeachment of Dilma Rousseff). Confluências / Revista Interdisciplinar de Sociologia e Direito, 22 (3), 172-197. Retrieved from https://periodicos.uff.br/confluencias/article/view/47121

Sepúlveda, N. (2018, November 19). La evidencia policial que confirmó el testimonio clave del menor que acompañaba al comunero Catrillanca (The police evidence that confirmed the key testimony of the minor who accompanied the community member Catrillanca). Ciper Chile. Retrieved from https://ciperchile.cl/2018/11/19/la-evidencia-policial-queconfirmo-el-testimonio-clave-del-menor-que-acompanaba-al-comunero-catrillanca/

Shima, J., Yoshida, M., \& Umemura, K. (2017, December). When do Users Change Their Profile Information on Twitter?. In 2017 IEEE International Conference on Big Data (Big Data) (pp. 3119-3122). IEEE. Retrieved from https:/doi.org/10.1109/BigData.2017.8258287

Smith, M., Rainie, L., Shneiderman, B., \& Hilmelboim, I. (2014). Mapping Twitter Topics Networks: From polarized crowds to community clusters. Numbers, facts and trends shaping the world. Pew Research Center. Retrieved from https://www.pewresearch.org/internet/2014/02/20/ mapping-twitter-topic-networks-from-polarized-crowds-to-community-clusters/

Stieglitz, S., Dang-Xuan, L., Bruns, A., \& Neuberger, C. (2014). Social Media Analytics: An Interdisciplinary Approach and Its Implications for Information Systems. Bus Inf Syst Eng, 6, 89-96. https://doi.org/10.1007/s12599-014-0315-7

Sunstein, C. R. (2002). The law of group polarization. The Journal of Political Philosophy, 10(2), 175-195. https://doi.org/10.1111/1467-9760.00148

Toledo Llancaqueo, V. (2007). Prima Ratio: Movilización mapuche y política penal. Los marcos de la política indígena en Chile 1990-2007 (Prima Ratio: Mapuche mobilization and penal policy. The frameworks of indigenous politics in Chile 1990-2007). OSAL 8(22). Retrieved from http://bibliotecavirtual.clacso.org.ar/ar/libros/osal/osal22/CDH22Toledo.pdf

Treré, E. \& Mattoni, A. (2016). Media ecologies and protest movements: main perspectives and key lessons. Information, Communication E Society, 19(3), 290-306. https://doi.org/10.1080/1369118X.2015.1109699

Tucker, J. A., Guess, A., Barberá, P., Vaccari, C., Siegel, A., Sanovich, S., \& Nyhan, B. (2018). Social media, political polarization, and political disinformation: A review of thescientific literature. William + Flora Hewlett Foundation. Retrieved from https://www.hewlett.org/wp-content/uploads/2018/ 03/Social-Media-Political-Polarization-and-Political-Disinformation-Literature-Review.pdf 
Uddin, M. M., Imran, M., \& Sajjad, H. (2014). Understanding types of users on Twitter. arXiv preprint arXiv:1406.1335. Retrieved from https://arxiv.org/abs/1406.1335

Uriel, E. \& Aldás, J. (2005). Análisis multivariante aplicado (Applied multivariate analysis). Madrid, Spain: Thomson.

Vaccari, C. (2018). Online Content and Political Polarization. In J. A. Tucker, A. Guess, P. Barberá, C. Vaccari, A. Siegel, S. Sanovich, S. Stuka, \& B. Nyhan (Eds.), Social Media, Political Polarization, and Political Disinformation: A Review of the Scientific Literature. William + Flora Hewlett Foundation. Retrieved from https://www.hewlett.org/ wp-content/uploads/2018/03/Social-Media-Political-Polarization-and-PoliticalDisinformation-Literature-Review.pdf

Vainio, J. \& Holmberg, K. (2017). Highly tweeted science articles: who tweets them? An analysis of Twitter user profile descriptions. Scientometrics, 112, 345-366. https://doi.org/10.1007/s11192-017-2368-0

\section{SOBRE LOS AUTORES}

MARCELO lUIS BARBosa dos SANTOS, Doctor en Ciencias de la Comunicación por la Pontificia Universidad Católica de Chile, Magíster en Comunicación y Semiótica por la Pontificia Universidad Católica de São Paulo. Investigador del Centro de Investigación y Documentación (CIDOC) de la Universidad Finis Terrae (UFT). Sus líneas de investigación emergen del cruce entre tecnologías digitales y democracia, incluyendo comunicación política y medios digitales, tecno-política y datificación de la sociedad, métodos digitales, entre otras. Es profesor de pregrado y postgrado en la escuela de Periodismo-UFT, donde coordina la mención en Comunicación Digital.

iD https://orcid.org/0000-0002-2658-3764

osCAR JARAMILLO CASTRO, Periodista de la Universidad Diego Portales (Chile) y Doctor en Ciencias de la Información de la Universidad Complutense de Madrid (España). Actualmente es Profesor Asociado de la Escuela de Periodismo de la Universidad Finis Terrae (Chile). Es investigador en el área del Análisis del Redes Sociales (ARS) y ciber ética.

iD https://orcid.org/0000-0001-9613-4836

DANIEL AGUIRRE AZócar, Profesor Asistente de la Universidad del Desarrollo (Chile), Facultad de Comunicaciones. Es también periodista por la University of Florida (EE.UU.), Master en Estudios Internacionales por la Universidad de Miami (EE.UU.) y Doctor en Ciencias de la Comunicación por la Pontificia Universidad Católica de Chile. Sus líneas de investigación abordan temáticas vinculadas a la comunicación política internacional y las tecnologías digitales.

iD https://orcid.org/0000-0002-3300-0088 